\title{
Cespace: sistema de fusión intersomática cervical. Estudio preliminar en 104 casos (120 implantes)
}

\author{
R. Arregui; J. Aso; J.V. Martínez-Quiñones; F. Consolini; N. Lambán* y M. Dominguez**
}

Servicio de Neurocirugía. Hospital MAZ (Zaragoza). *Servicio de Traumatologia. Hospital General Yagüe. Burgos. **Servicio de Neurocirugía Hospital Carlos Haya. Malaga.

\section{Resumen}

Cespace es un implante diseñado para conseguir la fusión intervertebral cervical sin injerto óseo. El implante está hecho de titanio y recubierto con plasmapore (polvo de titanio puro). El crecimiento óseo a través de la microestructura de titanio es inducido por el plasmapore, y la fusión se consigue progresivamente una vez que la inducción ósea ha terminado.

Desde Enero de 2002 a Diciembre de 2008 operamos a 104 pacientes empleando este implante en uno o dos niveles cervicales tras microdiscectomía radical. La patología más frecuente fue la radiculopatía causada por hernia discal o espondilosis $(\mathrm{N}=85 ; 81.7 \%)$, seguida de mielopatía $(\mathrm{N}=13 ; 12,5 \%)$. Seis casos $(5.7 \%)$ correspondían a fracturas cervicales o luxaciones. En estos últimos el sistema fue implantado como método de estabilización espinal combinado con placa de Caspar, evitando el injerto óseo.

El número total de implantes Cespace fue de $\mathbf{1 2 0 .}$

Los resultados clínicos fueron buenos en el $85.5 \%$ de los pacientes $(\mathrm{N}=89)$. La estabilidad primaria se obtuvo en todos los casos. La estabilidad secundaria (fusión) fue evidente después de 1 o 2 años en todos los implantes, confirmando la capacidad del plasmapore para la inducción de hueso sin injerto óseo.

No se registraron complicaciones específicas del implante (pseudoartrosis, hundimiento, inestabilidad, etc) en ningún caso.

Concluimos que la microdiscectomía radical y el implante con caja Cespace constituye un buen procedimiento para el tratamiento de la radiculopatía o mielopatía causadas por una hernia discal o espondilosis, evitando el injerto óseo y consiguiendo altas tasas de fusión cervical. En algunas fracturas cervicales asociadas a inestabilidad, el sistema Cespace puede ser usado como un sustituto fiable del injerto óseo intervertebral en combinación con placa de fijación anterior.

Recibido: 23-01-11. Aceptado: 23-02-11
PALABRAS CLAVE. Cespace. Microdiscectomía cervical. Fusión vertebral. Cajas de titanio. Plasmapore

Cespace. Cervical interbody fusion system. Preliminary retrospective study in 104 cases (120 implants)

Summary

Cespace is a cervical implant designed to obtain intervertebral fusion without bone grafting. The implant is built in titanium and coated with plasmapore (a sort of pure titanium powder). Bone growing through titanium microstructure is induced by the plasmapore, and fusion is progressively obtained once this boneinduction is completed.

From January 2002 to December 2008 we operated upon 104 patients employing this implant at one or two cervical spine levels. The more frequent condition was radiculopathy caused by disc herniation or spondilosis $(\mathrm{N}=85 ; 81.7 \%)$, followed by mielopathy $(\mathrm{N}=13 ; 12,5 \%)$. Six cases $(5.7 \%)$ corresponded to cervical fractures or dislocations. In these latter ones Cespace was implanted as a method of spinal stabilization combined with Caspar plates, avoiding bone grafting.

The total number of Cespace implants placed was 120.

Clinical results were good in $\mathbf{8 5 , 5 \%}$ of patients. Primary stability was obtained in all cases. Secondary stability (fusion) was evident after 1 to 2 years in all cases, confirming the bone-induction capability of plasmapore without bone grafting.

No specific implant complications (pseudoartroses, settling, instability, etc) were registered.

We conclude that radical microdiscectomy and Cespace box implant constitutes a good procedure for the treatment of cervical radiculopathy or mielopathy caused by disc herniation or spondylosis, avoiding bone grafting and providing high rates of vertebral fusion. In some cervical fractures associated to instability, Cespace can be used as a reliable substitute of intervertebral bone grafting in combination with anterior plate fixation. 
KEY WORDS. Cespace. Microdiscectomy cervical. Vertebral fusion. Titanium cages. Plasmapore

\section{Introducción}

En los últimos años, se han desarrollado distintos sistemas de fusión intersomática cervical para el tratamiento de las hernias discales, inestabilidades y fracturas. Entre ellos, un subgrupo de implantes, llamados cajas o jaulas, han conseguido un notable éxito.

Entre estos dispositivos, los hay que aprovechan la capacidad de algunos materiales para la inducción de formación de hueso. Estos implantes permiten alcanzar la fusión intervertebral sin necesidad de injerto óseo. Una nueva generación de cajas, proporciona, además estabilización primaria sin tornillos ni placas emergiendo como un "gold standard" en la fusión vertebral, al ofrecer tanto estabilidad primaria como secundaria.

En este trabajo exponemos nuestra experiencia después de un análisis retrospectivo de 104 casos de patología cervical tratados con uno de estos implantes: la caja "Cespace" (Aesculap). El total de implantes colocados fue de 120.

\section{Material y métodos}

\section{Casos}

Desde Enero de 2002 hasta Diciembre de 2008, 104 pacientes que padecían patología cervical fueron operados en nuestro Servicio usando este sistema. Se realiza un estudio retrospectivo de esta casuística.

Los pacientes se dividieron en dos grupos principales. Aquellos que presentaban afectación de raíces nerviosas o mielopatía causada por enfermedad discal o espondilosis, y los que sufrieron fracturas o luxaciones vertebrales. Los casos tratados y los niveles afectos se encuentran resumidos en las tablas 1 y 2. La tabla 3 muestra las características clínicas.

\section{Características del implante}

El sistema de fusión cervical anterior "Cespace" es un implante diseñado para proporcionar estabilidad tanto pri-

Tabla 1

Resumen de casos

\begin{tabular}{|l|c|c|c|c|c|}
\hline Pacientes & \multicolumn{3}{|c|}{ Edad (años) } \\
\hline Sexo & N & $\%$ & Media & Max. & Min. \\
\hline Hombres & 67 & 64,4 & 44 & 64 & 26 \\
\hline Mujeres & 37 & 35,6 & 42 & 63 & 32 \\
\hline
\end{tabular}

Tabla 2

Distribución de niveles afectados

\begin{tabular}{|l|r|r|}
\hline Nivel & $\mathbf{~ N}$ & $\mathbf{\%}$ \\
\hline C3-C4 & 1 & 0.8 \\
\hline C4-C5 & 3 & 2.4 \\
\hline C4-C5, C5-C6 & 3 & 2.4 \\
\hline C4-C5, C6-C7 & 1 & 0.8 \\
\hline C5-C6 & 45 & 36.3 \\
\hline C5-C6, C6-C7 & 12 & 9.7 \\
\hline C6-C7 & 35 & 28.2 \\
\hline C7-D1 & 4 & 3.2 \\
\hline Total & 104 & 100 \\
\hline
\end{tabular}

Tabla 3

Presentación clínica

\begin{tabular}{|l|r|r|}
\hline Cuadro clínico & N & \% \\
\hline Radiculopatía & 85 & 81.7 \\
\hline Mielopatía & 13 & 12.5 \\
\hline Inestabilidad (fracturas o luxaciones) & 6 & 5.7 \\
\hline Total & 104 & 100 \\
\hline
\end{tabular}

maria como secundaria (fusión) sin injerto óseo.

Fue concebido como un anillo sólido construido de titanio, cubierto con plasmapore. Esta última sustancia es, básicamente, un revestimiento con titanio puro que recubre el implante como una capa de polvo. Algunos trabajos han demostrado que el plasmapore induce la formación de hueso en el interior del implante, estimulando una integración sólida y real entre la superficie metálica de titanio y el cuerpo vertebral ${ }^{36,37,38}$. Se ha probado también que el plasmapore ofrece una resistente interfase frente a fuerzas tensiles y rotacionales ${ }^{29}$.

Un anillo de espículas, a modo de uña, corona la parte superior e inferior de las superficies de la caja, aportando estabilidad primaria como sistema de anclaje, una vez que han penetrado en el cuerpo vertebral.

El diseño lordótico de la caja garantiza el mantenimiento de la curvatura cervical.

La figura 1 representa la forma y morfología de Cespace. La tabla 4 representa la cantidad de implantes según el nivel de colocación.

\section{Tratamiento quirúrgico}

En todos los casos se realizó una discectomía micro- 


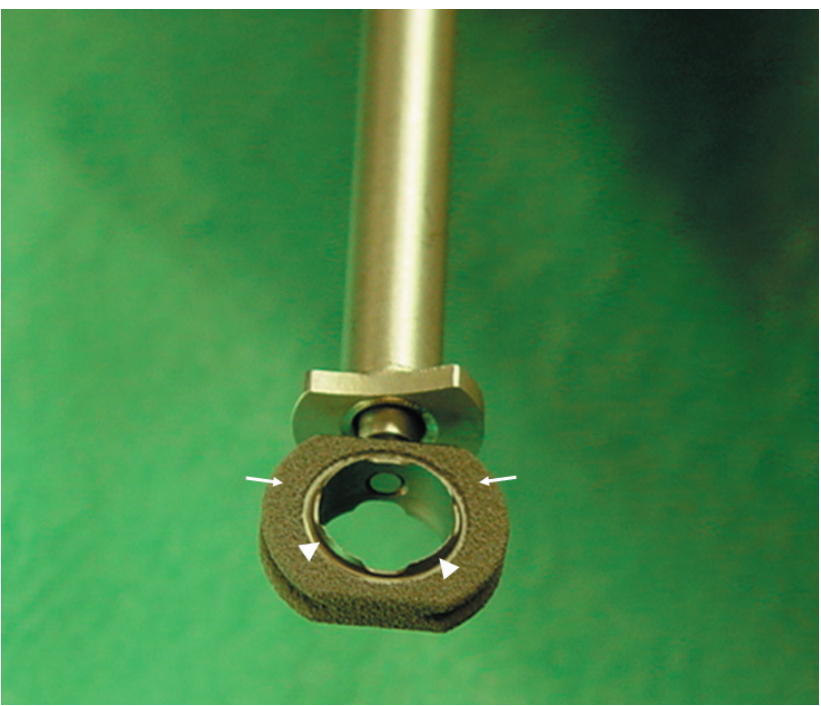

Figura 1. Aspecto del implante en donde puede apreciarse el recubrimiento de "plasmapore" (flechas), así como la corona que facilita el anclaje del mismo: estabilidad primaria (cabezas de flecha).

quirúrgica radical, incluyendo resección de osteofitos posteriores, cuando existían, y extracción de la eventual extrusión discal. El procedimiento fue llevado a cabo con microscopio, usando una fresa de alta velocidad e instrumentos delicados (pinzas de Kerrison de cabezal fino).

La discectomía incluyó limpieza de los platillos vertebrales eliminando el disco remanente y, en ocasiones, fresando con sumo cuidado las superficies óseas, para conseguir un completo contacto entre el plasmapore y la
Tabla 4

Distribución de los implantes

\begin{tabular}{|l|r|c|}
\hline Nivel & $\mathbf{N}$ & $\mathbf{\%}$ \\
\hline C3-C4 & 1 & 0,8 \\
\hline C4-C5 & 7 & 5,8 \\
\hline C5-C6 & 60 & 50 \\
\hline C6-C7 & 48 & 40 \\
\hline C7-D1 & 4 & 3,3 \\
\hline Total & 120 & 100 \\
\hline
\end{tabular}

lámina externa de las vértebras. El ligamento común posterior fue abierto transversal y ampliamente en todos los casos, hasta exponer la duramadre. (Figura 2).

En todos los casos la caja fue introducida bajo distracción (3-5 Kg aplicada a la cabeza mediante fronda de Glisson).

Las dimensiones de la caja "Cespace" fueron probadas usando una herramienta de medida incluida en el set de colocación del implante.

Una vez colocada la caja, se retiró la distracción, permitiendo a ambos cuerpos vertebrales comprimir progresivamente el implante, al objeto de lograr un adecuado anclaje de la corona de espículas en cada platillo, superior e inferior.

La colocación del implante se controló mediante radioscopia intraoperatoria estática y en flexo-extensión. (Figuras 3 y 4 ).

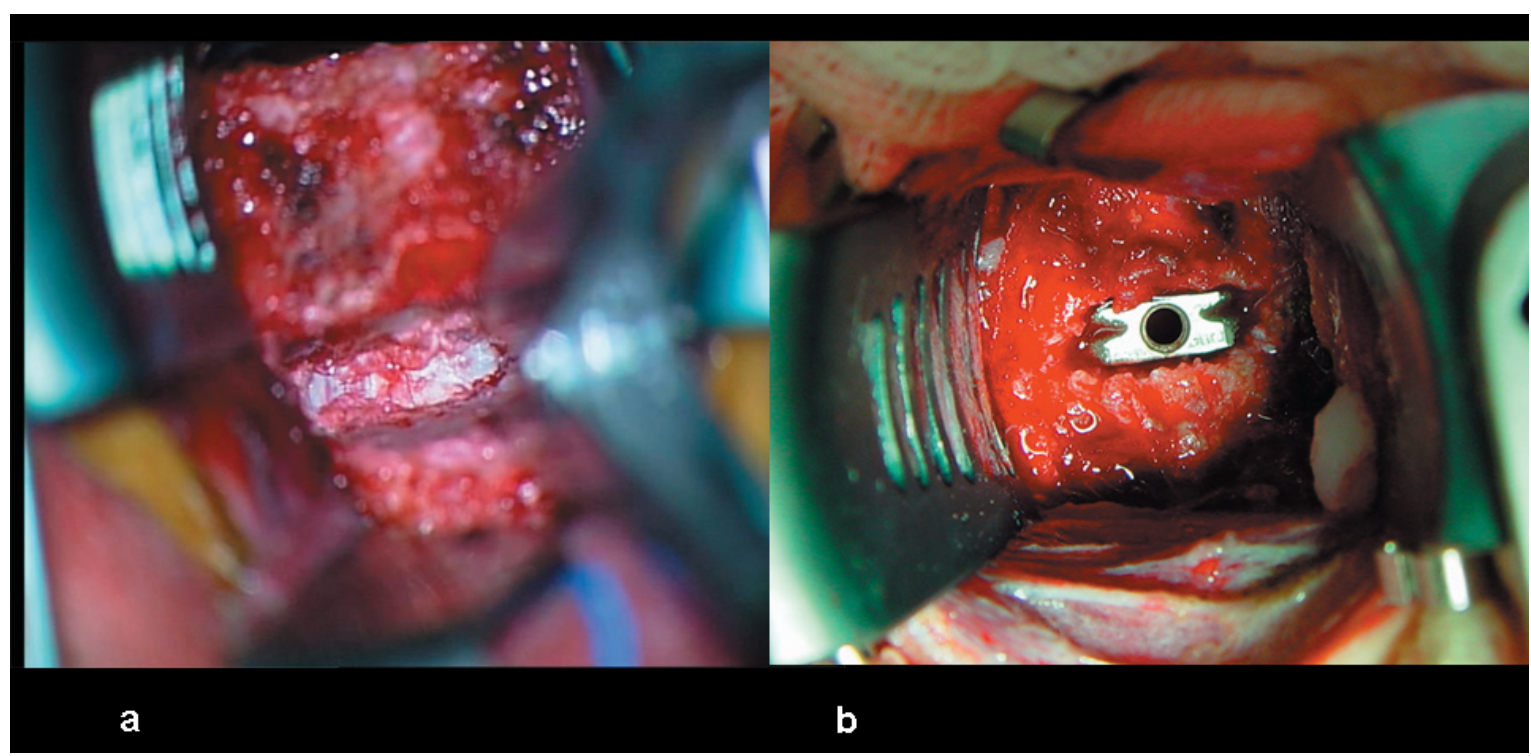

Figura 2. Imagen operatoria de la colocación de una caja cespace. En "a" puede apreciarse la realización de una microdiscectomía radical, con apertura completa del ligamento vertebral común posterior. En " $b$ " puede verse el implante en su emplazamiento definitivo. 


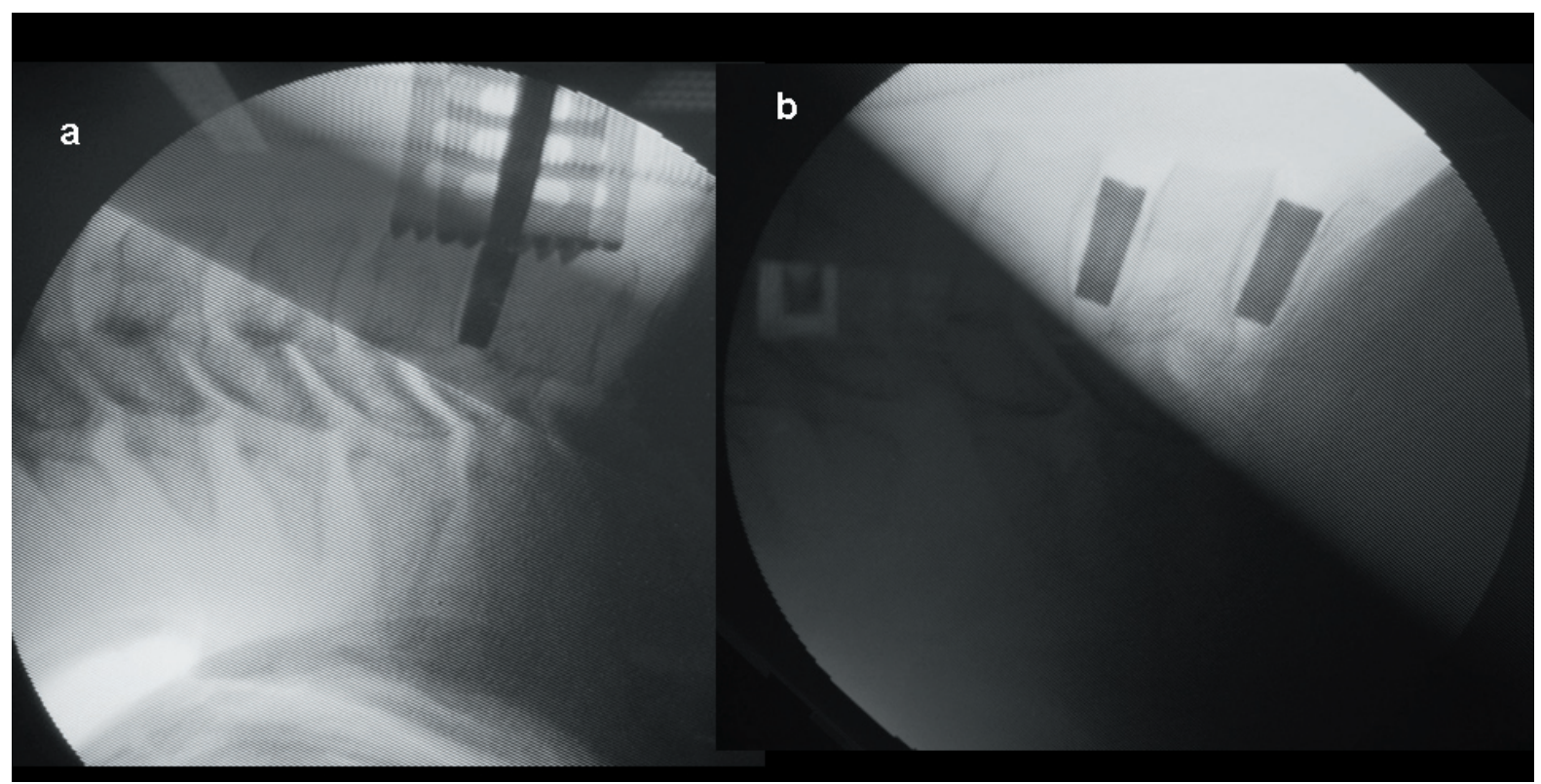

Figura 3. Fotografía mediante radioscopia intraoperatoria. En "a" se aprecia la introducción del implante y en "b" el aspecto tras la colocación de dos cajas Cespace. El control radiológico intraoperatorio se realiza habitualmente en posición neutra, flexión y extensión, a fin de garantizar la ausencia de movilidad del mismo.

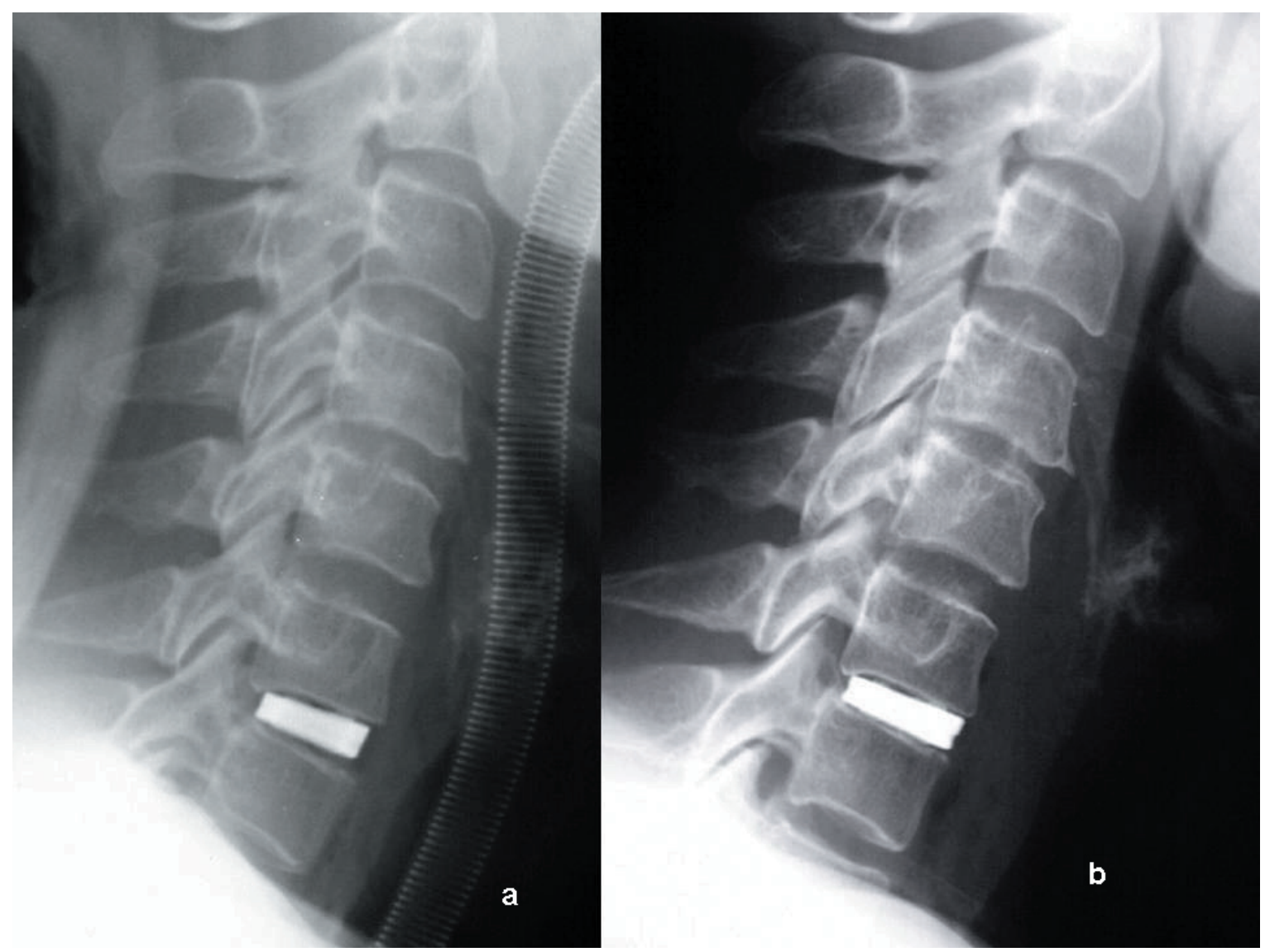

Figura 4. La imagen " a" corresponde a una radiografia intraoperatoria con distracción, tras colocación de la caja. La figura "b", tomada una semana más tarde, muestra el colapso del espacio platillo vertebral-implante, tanto por encima como por debajo de la prótesis. Ello indica una adecuada estabilización primaria, al entrar la corona de especulas dentro del platillo vertebral. 


\section{Cuidados postoperatorios y seguimiento}

Los cuidados postoperatorios incluyeron un collar blando durante tres días. Los pacientes fueron dados de alta hospitalaria a los tres días de la cirugía.

El seguimiento incluyó una revisión clínica a las cuatro semanas después de la cirugía, y visitas mensuales hasta el sexto mes. Todos los pacientes fueron seguidos como mínimo hasta el año de la cirugía, sin registrarse pérdidas de seguimiento en ningún caso.

La vuelta al trabajo se permitió entre los tres y seis meses después de la cirugía en la mayoría de los casos. Las radiografías de control se realizaron mensualmente durante los primeros dos meses, y posteriormente al sexto mes y al año. El seguimiento radiológico se prolongó hasta los tres años, en 92 pacientes.

La valoración del estado clínico se realizó mediante el sistema preconizado por Herron ${ }^{13}$. Si bien fue diseñado para la región lumbar, puede ser aplicado a la cervical. Tiene la ventaja de considerar aspectos como la reincorporación laboral, parámetro que en nuestro medio resulta importante al tratarse en muchos casos de accidentes laborales.

Nosotros no consideramos el TC como un método adecuado para control de la fusión en nuestros casos. No encontramos utilidad de esta exploración excepto en la valoración del crecimiento excesivo de hueso. Por lo tanto el TC no se incluyó en el protocolo de seguimiento.

La RMN fue realizada sólo en pacientes con síntomas neurológicos en el postoperatorio o en casos con sospecha de síndrome del segmento adyacente.

\section{Resultados}

Dividimos el resultado en tres categorías: situación clínica, fusión y complicaciones.

\section{Situación clínica}

El estado clínico fue evaluado basándonos en el "sistema de puntuación del resultado quirúrgico para laminectomía y discectomía", adaptado a la región cervical $^{13}$ (Tabla 5). La tabla 6, muestra los resultados clínicos resumidos.
Tabla 6

Resultados clínicos a los seis meses de la cirugía

\begin{tabular}{|l|r|r|}
\hline Resultados & N & \% \\
\hline Bueno & 89 & 85,5 \\
\hline Regular & 9 & 8,6 \\
\hline Malo & 6 & 5,7 \\
\hline Total & 104 & 100 \\
\hline
\end{tabular}

\section{Estabilidad primaria}

No se observaron desplazamientos de las cajas en ninguno de nuestros pacientes. Las medidas fueron tomadas sobre archivos DICOM para evaluar los posibles desplazamientos de la caja en $\mathrm{Rx}$, en radiografías tanto estáticas como dinámicas en todos los pacientes, sin comprobar desplazamientos anteriores o laterales.

\section{Fusión}

Fue difícil sacar conclusiones definitivas sobre la fusión, debido a la falta de acuerdo en la literatura sobre el criterio definitivo para los diferentes implantes de columna cervical ${ }^{35}$. Por analogía, algunas revisiones han indicado algunos criterios de fusión, entre los que se encuentran los siguientes ${ }^{18}$ : ausencia de movimiento en radiografías en flexión y extensión; ausencia de halo alrededor del implante. Cohesión completa y desaparición de la interfase entre platillo e implante; presencia bilateral de hueso trabecular continuo entre los segmentos fusionados.

El primer criterio fue comprobado en nuestros pacientes con Rx dinámicas, no mostrando desplazamientos significativos en ninguno de ellos. Sólo un paciente tuvo un aumento leve del interespacio a lo largo de la interlínea superior (entre el cuerpo y el implante) en las imágenes tomadas el segundo mes en flexo-extensión, pero este hallazgo desapareció en controles posteriores.

Ningún caso mostró halo alrededor del implante. En

Tabla 5

\section{Clasificación de los resultados}

\begin{tabular}{|l|l|}
\hline Bueno & $\begin{array}{l}\text { Paciente que regresa a su ocupación anterior o similar, no molestias, no analgésicos o uso ocasional de } \\
\text { analgésicos menores, no déficit motores }\end{array}$ \\
\hline Regular & $\begin{array}{l}\text { Paciente que tiene que cambiar de ocupación y hacer reposo, control del dolor con analgésicos menores, } \\
\text { mejoría parcial del dolor con la actividad, resolución parcial del déficit neurológico. }\end{array}$ \\
\hline Malo & $\begin{array}{l}\text { Paciente incapaz de regresar al trabajo, mejoría parcial del dolor, necesidad ocasional de analgésicos } \\
\text { mayores, no modificación del déficit neurológico. }\end{array}$ \\
\hline
\end{tabular}




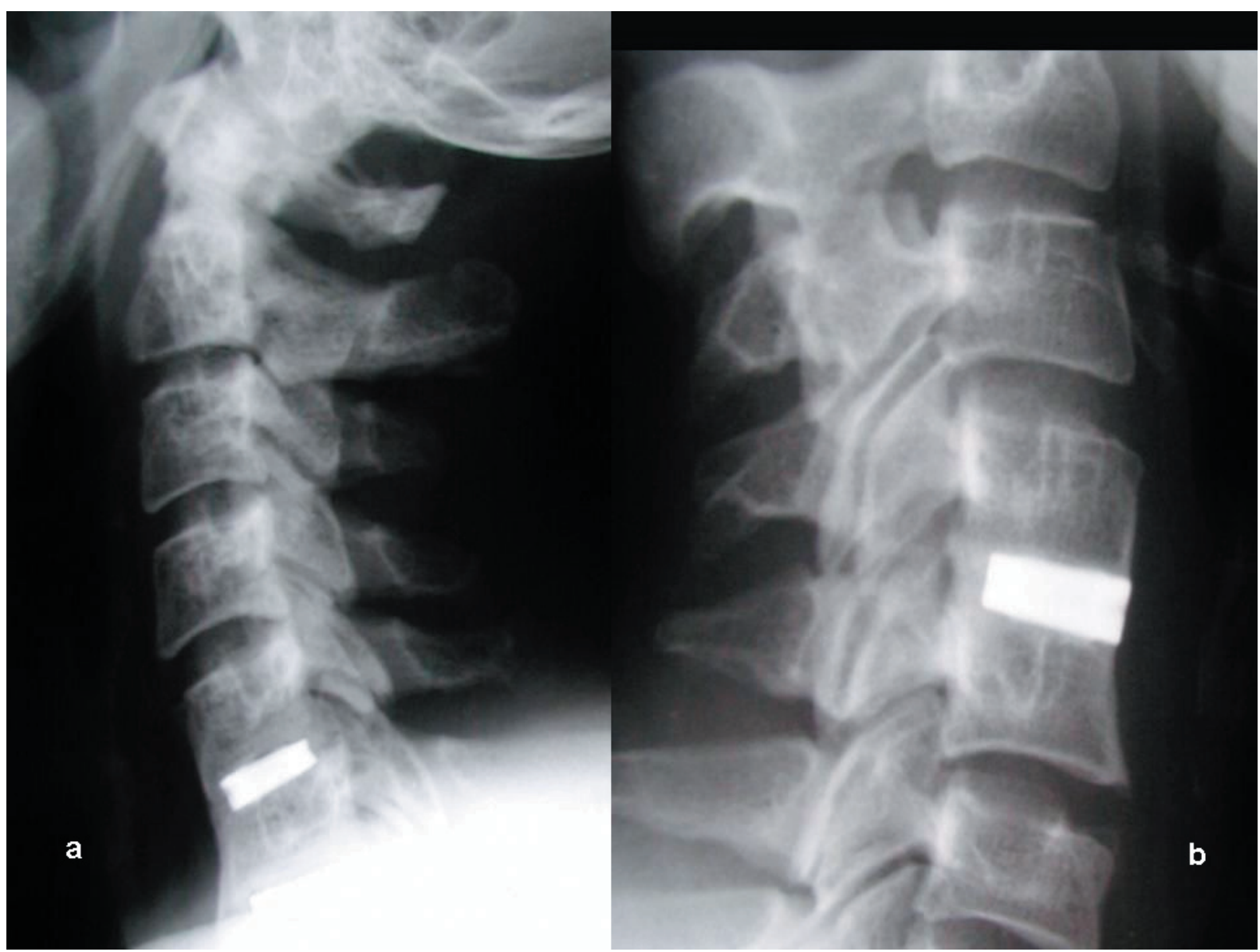

Figura 5. Imágenes de estabilización secundaria (fusion). En "a", correspondiente a un examen practicado a los dos años de la cirugía, se observa el crecimiento de hueso encastrando literalmente el implante superior y parte del inferior. En "b" se aprecia el borramiento de la interfase implante-platillos (signo precoz de integración), así como crecimiento óseo posterior. Esta ultima imagen fue tomada a los 13 meses de la cirugía.

lugar de esto, la interlínea observable inicialmente entre el implante y el cuerpo tendió a colapsarse. Entre el tercer y sexto mes se apreció un colapso de la interlínea, con difuminado de la interfase hueso-implante. La Tabla 7 representa los cambios observados en las radiografías y el intervalo de tiempo en nuestros casos.

El tercer criterio (presencia bilateral de hueso trabecular continuo entre los segmentos) se aprecia con dificultad, en controles radiológicos, en este tipo de implantes antes de los seis meses o, incluso, hasta el año después de la cirugía, siendo evidente a partir de esta fecha en la mayoría de los casos. (Figura 5).

Los resultados de estos signos de estabilidad secundaria, figuran en la Tabla 7.

Tabla 7

Fases de integración del implante óseo (Rx simple)

\begin{tabular}{|l|c|c|c|c|c|c|}
\hline \multirow{2}{*}{ Criterios } & \multicolumn{2}{|c|}{$\mathbf{6}^{\mathbf{0}}$ mes } & \multicolumn{2}{|c|}{$\mathbf{1}$ ã̃o } & \multicolumn{2}{|c|}{$\mathbf{3}$ años } \\
\cline { 2 - 7 } & $\mathbf{n}$ & $\mathbf{\%}$ & $\mathbf{n}$ & $\mathbf{\%}$ & $\mathbf{n}$ & $\mathbf{\%}$ \\
\hline Ausencia de movilidad del segmento, valorado en Rx dinámicas laterales & 114 & 100 & 114 & 100 & 92 & 100 \\
\hline Cohesión completa y desaparición de la interfase entre platillo e implante & 82 & 71,9 & 95 & 83,3 & 79 & 85,5 \\
\hline Puentes de unión intervertebrales & 66 & 57,8 & 103 & 90,3 & 85 & 92,3 \\
\hline
\end{tabular}




\section{Tabla 8}

Complicaciones

\begin{tabular}{|l|c|}
\hline \multicolumn{1}{|c|}{ Complicaciones } & Casos \\
\hline Penetración de la caja en el cuerpo vertebral & 0 \\
\hline Movilización/Extrusión del implante & 0 \\
\hline Pseudoartrosis & 0 \\
\hline Inestabilidad & 0 \\
\hline Cifosis & 3 \\
\hline Osteofito posterior, estrechando el agujero radicular & 2 \\
\hline Luxación del implante & 0 \\
\hline Abceso & 1 \\
\hline
\end{tabular}

\section{Complicaciones}

La tabla 8 resume las complicaciones y efectos adversos observados en nuestros pacientes, referido a los efectos adversos o complicaciones descritos en la literatura.

\section{Discusión}

En los últimos años, se han desarrollado implantes intersomáticos cervicales para ofrecer un modo definitivo de conseguir una estabilidad y fusión vertebral después de la discectomía o corpectomía. Entre estos dispositivos, las cajas han alcanzado una popularidad significativa ${ }^{1,23}$.

Existen varios modelos, con diferentes materiales y formas ${ }^{6,7,31}$ que persiguen, entre otros objetivos, conseguir la fusión de las vértebras adyacentes y mantener la altura del interespacio discal.

Hasta hace no mucho tiempo, las cajas disponibles, requerían, para lograr la fusión, el empleo adicional de injerto óseo dentro del dispositivo ${ }^{44}$. Sin embargo, la utilización de hueso homólogo o heterólogo no está exenta de problemas ${ }^{5,33}$. Además, el hueso autólogo esponjoso, como relleno de las cajas, proporciona una resistencia inicialmente débil ${ }^{15}$.

Por otro lado, el material estándar para la fusión vertebral, el hueso tricortical, empleado en los sistemas placainjerto, es de integración lenta. Tampoco el hueso de banco ha demostrado una alta capacidad para la estimulación de inducción ósea ${ }^{12,42}$.

Por tanto, los sistemas similares al Cespace podrían representar una alternativa a la fusión con injerto en el raquis cervical.

La evolución de nuestros casos puede discutirse de tres modos: situación clínica, evolución mecánica (estabilidad primaria), estabilidad secundaria (fusión vertebral).

\section{Situación clínica}

Los resultados clínicos de todos nuestros pacientes fueron buenos en el 85,5\%, y malos sólo en el 5.7\%. El sistema de puntuación clínica fue el de Herron et a ${ }^{13}$, aplicado al raquis cervical. Este sistema proporciona una buena idea de la evolución clínica pues contempla los resultados para el trabajo. Muchos de nuestros casos fueron accidentes laborales (hernias de disco cervicales que en España tienen la consideración de accidente laboral cuando el cuadro se inicia en el trabajo), por lo que este sistema resulta útil al tener en cuenta quiénes han podido reanudar sus actividades laborales habituales.

Sin embargo, la evolución clínica satisfactoria podría deberse no sólo a las propiedades de los implantes, sino también a la técnica quirúrgica empleada, que incluyó una cuidadosa descompresión de la raíz nerviosa.

La microdiscectomía radical y microdescompresión nerviosa probablemente constituyen el estándar más importante del procedimiento quirúrgico $\mathrm{o}^{30}$, $\mathrm{y}$ representan el principal factor del alivio del dolor en estos casos.

En cambio, la microdiscectomía sola, quizás no es el único factor que explica nuestros resultados. Después de comparar la microdiscectomía sola, la fusión con autoinjerto, interposición de polimetilmetracrilato, y las cajas de titanio (TTC), Barlocher encontró mejores resultados con TTC en el dolor radicular y el periodo de vuelta al trabajo (92\% excelentes y buenos resultados seis meses después de la cirugía) ${ }^{3}$. Es posible que el restablecimiento de la altura normal del disco y de las dimensiones del foramen contribuyan también a proporcionar un buen resultado clínico. Igualmente, como veremos a continuación, este sistema proporciona una sólida estabilización primaria y secundaria, lo que puede actuar mejorando el dolor segmentario y radicular. Estos objetivos pueden conseguirse con sistemas placa-injerto, pero en general conllevan más rigidez y mayor interposición de material foráneo.

Los pacientes que presentaron un resultado calificado como malo, fueron accidentes laborales en su totalidad. Dos pacientes presentaron signos mielopáticos de suficiente entidad como para impedir la vuelta al trabajo. En el resto, pudieron interferir variables laborales o médico-legales (estilo de respuesta distorsionado o no fidedigno por expectativas de ganancia primaria, secundaria o terciaria) ${ }^{2}$.

\section{Estabilidad primaria}

En nuestros pacientes no observamos desplazamiento de la caja en las radiografías estáticas o dinámicas en ningún caso. Creemos que el periodo de seguimiento (cercano a los seis años en algunos casos) es suficiente para confirmar la excelente estabilidad primaria proporcionada por este sistema.

No medimos en nuestros casos la estabilidad rotacional ni en inclinación lateral. Sin embargo, trabajos previos indican que las cajas cilíndricas son las más eficientes en 


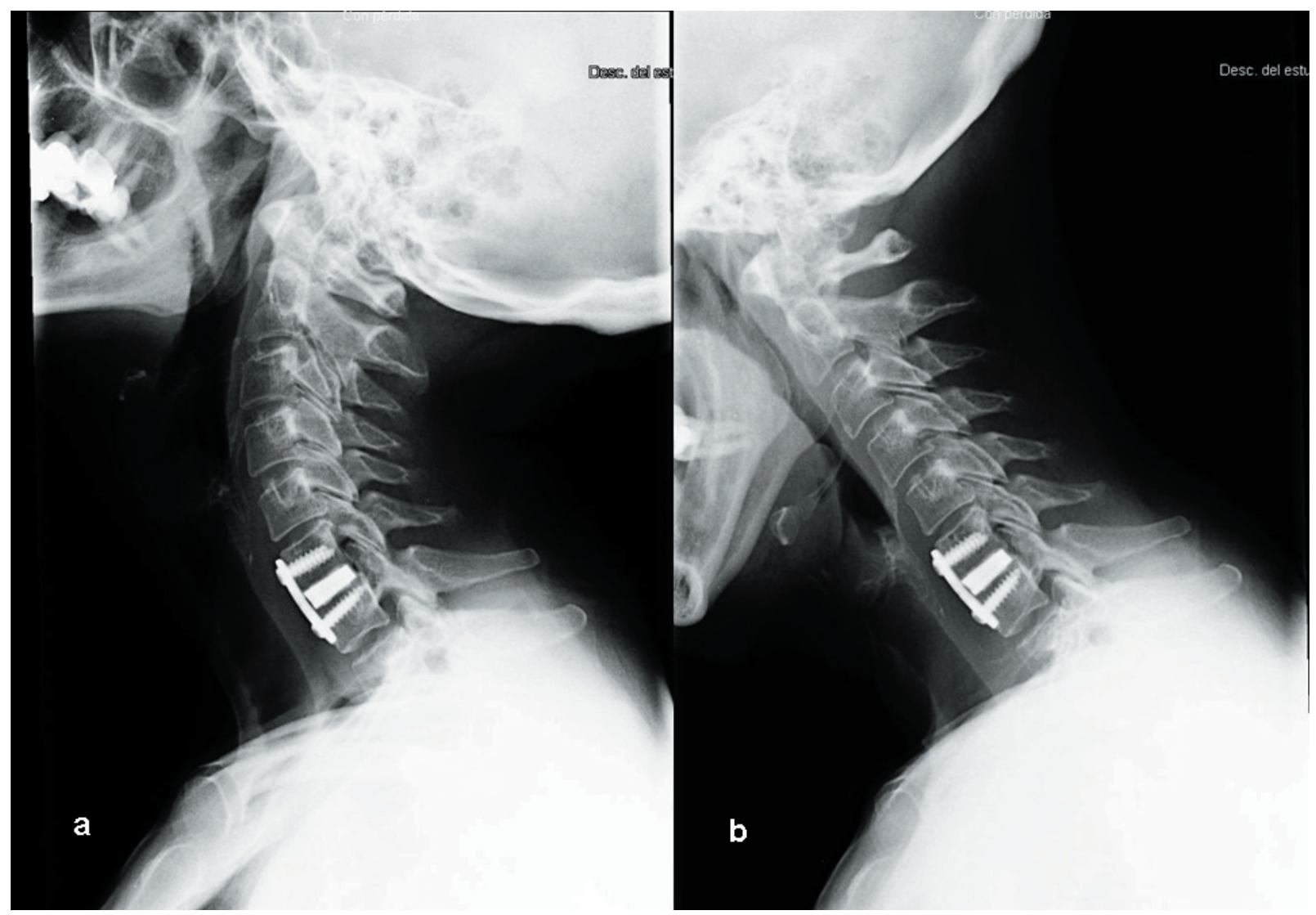

Figura 6. Ejemplo de tratamiento de una luxación vertebral mediante caja Cespace y placa de Caspar. Dadas las excelentes posibilidades de estabilización tanto primaria como secundaria de la caja, resulta un sistema ideal para combinarlo con placas atornilladas, evitando injerto autólogo y con muy buenos resultados biomecánicos. La imagen "a" fue tomada en extensión y la "b" en flexión.

controlar los movimientos de extensión e inclinación late$\mathrm{ral}^{16}$. Nuestra serie indica, por tanto, que el sistema Cespace proporciona un bloqueo temprano del segmento operado (estabilización primaria).

\section{Estabilidad secundaria (fusión vertebral)}

Algunos trabajos han demostrado buenos resultados en fusión vertebral utilizando dispositivos de titanio. Sin embargo, la mayoría se basa en el empleo de cajas o mallas de titanio rellenas de hueso $0^{1,20,21,24,39}$. En estas series el resultado de fusión obtenido fue superior al 90\%. Sin embargo, hay menos experiencia con los dispositivos recubiertos de plasmapore, sin relleno de injerto óseo.

Algunos estudios llevados a cabo en prótesis óseas articulares fuera de la columna vertebral han demostrado, que implantes no cementados con superficies recubiertas de titanio, consiguen buenos resultados, demostrando una

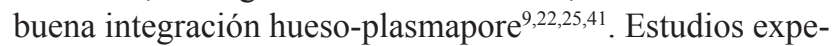
rimentales prueban que la fusión intersomática vertebral es posible empleando titanio cubierto de plasmapore sin injerto óseo $^{1}$. Otros trabajos han confirmado histológicamente el crecimiento de hueso a través del titanio ${ }^{19}$.

Una desventaja de los sistemas recubiertos de titanio es, sin embargo, la dificultad para determinar la fusión ${ }^{32} . \mathrm{Si}$ usamos el criterio radiológico más evidente para la fusión, como es la presencia de puentes óseos entre las vértebras adyacentes, en nuestra serie, la tasa de fusión es elevada (66,3\% al sexto mes, $91 \%$ en el primer año).

\section{Otras consideraciones}

Una cuestión adicional e interesante es el uso de Cespace, combinado con placa atornillada, en un segmento inestable a consecuencia de una fractura o luxación vertebral. Aunque nuestra experiencia es limitada (6 casos), su uso se ha confirmado como un sustituto de injerto óseo seguro y fiable ${ }^{17}$, ya que en todos ellos se logró una completa estabilidad secundaria (figura 6).

Otra posibilidad es el empleo de la caja en combinación con prótesis articulada. La figura 7 muestra uno de estos casos. Esta combinación puede ser beneficiosa en pacientes jóvenes con afectación discal múltiple para evitar el síndrome del segmento adyacente. 


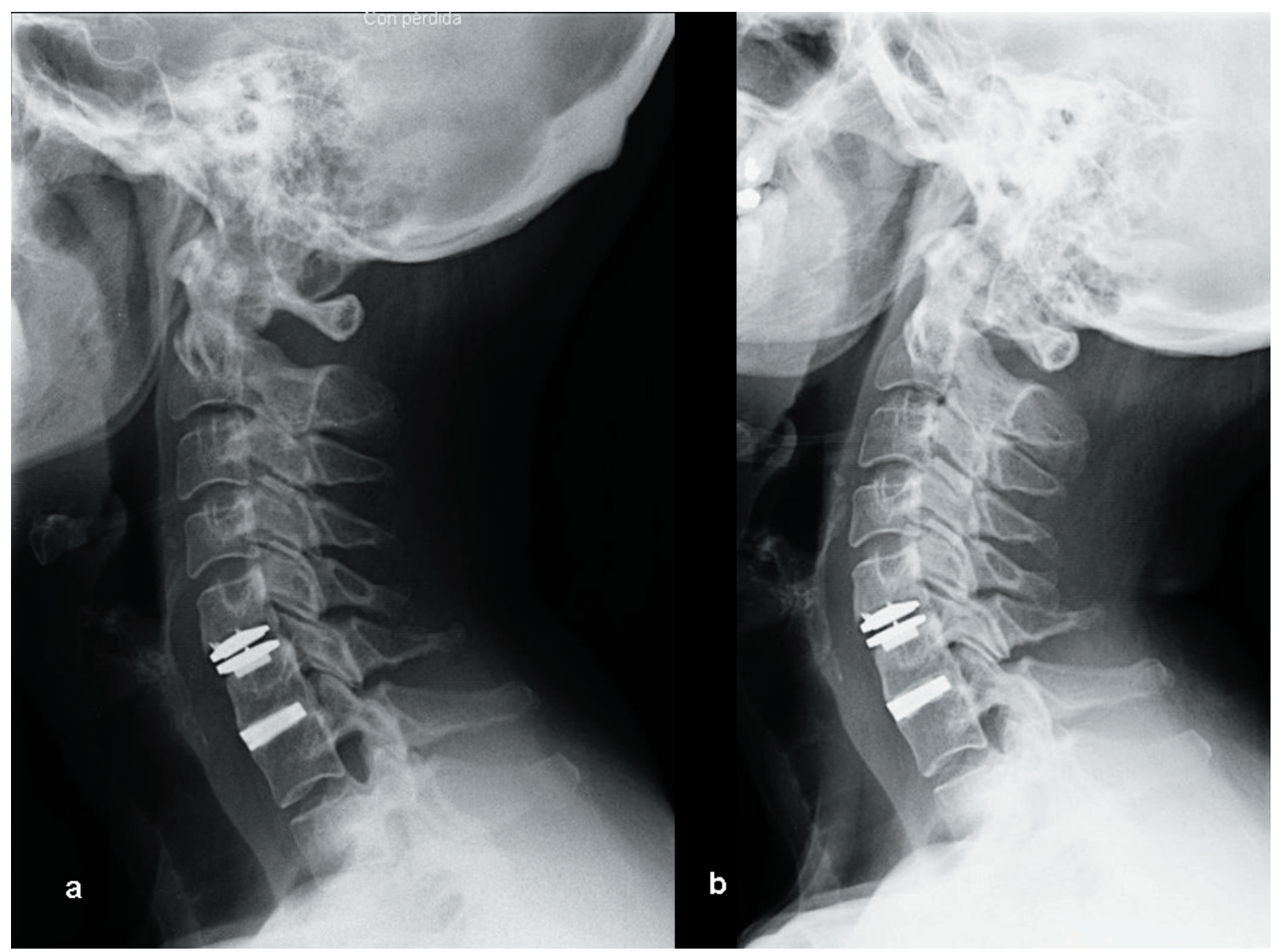

Figura 7. Imagen de combinación caja-prótesis articulada (Cespace-ActivC). Esta modalidad permite lograr la fusión de un segmento marcadamente deteriorado, evitando el desarrollo de síndrome del segmento adyacente en otro espacio afecto también por la degeneración disco-vertebral en menor medida, en especial en personas jóvenes. Puede apreciarse en ambas imágenes el borramiento de la interfase platillo-Cespace y el incipiente crecimiento de hueso enmarcando la caja. En "a" (flexión) y en " $b$ " (extensión), se aprecia una buena movilidad de la prótesis articulada y una fusión del espacio inferior.

Hemos encontrado una ventaja adicional en el sistema Cespace, la reducción del tiempo quirúrgico. El sistema no requiere placas ni tornillos para conseguir la estabilización primaria, reduciendo considerablemente el tiempo de cirugía.

Un estudio extenso en sistemas de espondilodesis graves cervicales indica que el sistema Cespace presenta un fácil manejo y costo moderado ${ }^{26,27}$.

\section{Complicaciones}

Algunas complicaciones, relacionadas con la estabilidad primaria en los implantes cervicales, incluye el hundimiento en el cuerpo vertebral, extrusión del implante, pseudoartrosis, inestabilidad, cifosis, luxación del implante.

El hundimiento puede ser definido como el descenso en la altura total vertical de las dos vértebras fusionadas medido en la Rx lateral realizada 3 y 6 meses postoperativamente comparada con las radiografías inmediatamente postoperatorias ${ }^{40}$. Van Jonbergen encontró una alta tendencia en 125 pacientes operados con cajas de titanio, indicando que mejoras en el diseño de las cajas permitiendo un mayor o mas extenso contacto con los platillos podrían evitar este fenómeno. Otros trabajos no han encontrado que cause problemas clínicos o altere el mecanismo de fusión ${ }^{4,34}$. En nuestra serie no encontramos ningún caso con esta complicación. Es posible que el tratamiento dado a los platillos vertebrales (mínimo refresco o erosión) permitido por el abordaje microquirúrgico, así como el diseño de la placa que permite un amplio contacto con la cortical ósea de ambos platillos, contribuya a la nula incidencia de estas complicaciones.

Otro tipo de complicaciones son inherentes a la fusión en sí misma, también llamada "Síndrome del segmento adyacente". El síndrome del segmento adyacente es observado como un suceso que ocurre sea cual sea el proceso de fusión empleado ${ }^{14,43}$. Este hecho parece inevitable tras un proceso de fusión y constituye quizá la mayor de las desventajas frente a sistemas como la artroplastia cervical, que mantienen cierta movilidad del segmento reemplazado ${ }^{10}$.

Las complicaciones generales de la cirugía son también 
posibles. En nuestros casos, un paciente desarrolló un absceso cervical que requirió tratamiento quirúrgico, mediante drenaje y lavado antiséptico, con una evolución excelente sin necesidad de retirar el implante.

Un inconveniente significativo son los artefactos en la RNM producidos por los dispositivos de titanio, lo que dificulta la valoración de los segmentos intervenidos. Sin embargo, una intensidad de campo menor y el uso de técnicas spin-eco rápidas pueden reducir los artefactos de este tipo $^{8,28}$.

Factores como la corrosión, formación de detritus metálicos y producción de productos de corrosión potencialmente tóxicos para el paciente, constituyen otros problemas en los implantes espinales. Sin embargo, algunos trabajos sobre la artroplastia discal mantienen que la capa de titanio es la más adecuada para el uso ${ }^{11}$. Son precisos, por tanto, seguimientos mas prolongados de los sistemas de artroplastia para valorar estas potenciales complicaciones de los materiales a largo plazo. En tal sentido, la artroplastia ofrece ventajas biomecánicas a los sistemas de fusión, pero constan de materiales diversos cuyo comportamiento a largo plazo es todavía insuficientemente conocido.

\section{Conclusiones}

Los resultados de nuestros pacientes indican que el sistema de fusión anterior cervical Cespace es un método fiable, seguro y fácil para conseguir una fusión de cuerpos cervicales en la enfermedad discal.

Las cajas recubiertas de titanio constituyen un "gold standard" en la fusión cervical sin injerto óseo. En asociación con una cuidadosa técnica micro-neuroquirúrgica, permiten conseguir los objetivos de descompresión neural, estabilización primaria y fusión ósea.

El sistema Cespace podría ser usado como sustituto del injerto óseo en el segmento cervical inestable como consecuencia de fracturas o luxaciones. Sin embargo, nuestra experiencia al respecto es limitada y se requieren casuísticas mayores para establecer conclusiones definitivas. ${ }^{*}$

\section{Bibliografia}

1. Al-Hami, S.: Cervical monosegmental interbody fusion using titanium implants in degenerative, intervertebral disc disease. Minim Invasive Neurosurg1999; 42: 10-17.

2. Aso, J., Martínez Quiñones, J.V., Arregui Calvo, R.: Simulación en columna vertebral. Grupo 2 Editores. Madrid 2005

3. Barlocher, C.B., Barth, A., Krauss, J.K., Binggeli, R., Seiler, R.W.: Comparative evaluation of microdiscectomy

\footnotetext{
* No hay conflicto de intereses con ninguno de los firmantes del trabajo.
}

only, autograft fusion, polymethylmethacrylate interposition, and threaded titanium cage fusion for treatment of single-level cervical disc disease: a prospective randomized study in 125 patients.

4. Bartels, R.H,. Donk, RD., Feuth, T.: Subsidence of stand-alone cervical carbon fiber cages. Neurosurgery. 2006; 58: 502-508.

5. Bishop, R.C., Moore, K.A., Hadley, M.N.: Anterior cervical interbody fusion using autogeneic and allogeneic bone graft substrate: a prospective comparative analysis. J Neurosurg 1996; 85: 206-210.

6. Brantigan, J.W., Steffee, A.D.: A carbon fiber implant to aid inter-body lumbar fusion. Two-year clinical results in the first 26 patients. Spine 1993; 18: 2106-2107.

7. Chi-Chien, N., Lih-Huei, C.H., Po-Liang, L., TsaiSheng, F., Wen-Jer, C.H.: Trapezoidal Titanium Cage in Anterior Cervical Interbody Fusion: A Clinical Experience Chang Gung Med J 2005; 28: 212-221.

8. Farahani, K., Sinha, U., Sinha, S.: Effect of field strength on susceptibility artifacts in magnetic resonance imaging. Comput Med Imaging Graph 1990; 14: 409-413.

9. Friedman, R.J., A.N., Y.H., Ming, J., Draughn., R.A, Bauer., T.W.: Influence of biomaterial surface texture on bone ingrowth in the rabbit femur. Journal of Orthopaedic Research, 1996; 14: 455-464.

10. Goffin, J., Geusens, E., Vantomme, N., Quintens, E., Waerzeggers,Y., Depreitere, B., Van Calenbergh, F., Van Loon, J.: Long-term follow-up after interbody fusion of the cervical spine. J Spinal Disord Tech. 2004; 17: 79-85.

11. Hallab, Nadim., Link, Helmut, D., Mcafee, P.C., Biomaterial Optimization in Total Disc Arthroplasty.. Spine. 2003; 28: S139-S152.

12. Hartwig, C.H., Esenwein, S.A., Pfund, A., Kusswetter Dagger, W., Herr, G.: Improved osseointegration of titanium implants of different surface characteristics by the use of bone morphogenetic protein (BMP-3): an animal study performed at the metaphyseal bone bed in dogs. Z Orthop Ihre Grenzgeb. 2003; 141: 705-711

13. Herron, L.D., Turner, J.: Patient selection for lumbar laminectomy and discectomy with a revised objective rating system. Clin Orthopaedics Related Res. 1985; 199: 145152.

14. Hilibrand, A.S., Carlson, G.D., Palumbo, M.A., Jones, P.K., Bohlman, H.H., Radiculopathy and myelopathy at segments adjacent to the site of a previous anterior cervical arthrodesis. J Bone Joint Surg Am. 1999; 81:519-528.

15. Kanayama, M., Hashimoto, T., Shigenobu, K., Oha, F., Ishida, T., Yamane, S.: Pitfalls of anterior cervical fusion using titanium mesh and local autograft. J Spinal Disord 2003; 16: 513-518.

16. Kandziora, F., Pflugmacher, R., Schafer, J., Born, C., Duda, G., Haas, N.P., Mittlmeier, T.: Biomechanical comparison of cervical spine interbody fusion cages. Spine 2001; 26: 
$1850-1857$.

17. Kandziora, F., Pflugmacher, R., Scholz, M., Schnake, K., Putzier, M., Khodadady, A.N., Klostermann, C., Haas, N.P.: Treatment of traumatic cervical spine instability with interbody fusion cages: a prospective controlled study with a 2-year follow-up. Injury. 2005; 36 , 2: B27-35.

18. La Rosa, G., Cacciola Conti, A., Cardali, S., La Torre, D., Gambadauro, N.M., Tomasello, F.: Posterior fusion compared with posterior interbody fusion in segmental spinal fixation for adult spondylolisthesis Neurosurg Focus 2001; $10: 9$

19. Lange, M., Philipp, A., Fink, U.: Anterior cervical spine fusion using RABEA-Titan-Cages avoiding iliac crest spongiosa: first experiences and results. Neurol Neurochir 2000; 64-69.

20. Leong, J.C., Chow, SP., Yau, A.C.: Titanium-mesh block replacement of the intervertebral disk. Clin Orthop 1994; 52-63.

21. Majd, M.E., Vadhva, M., Holt, R.T.: Anterior cervical reconstruction using titanium cages with anterior plating. Spine 1999; 1; 24: 1604-1610.

22. Martin, J.Y., Schwartz, Z., Hummert, T.W., Schraub, D.M., Simpson, J., Lankfordj, J.R., Dean, D.D., Cochran, D.L., Boyan, B.D.: Effect of titanium surface roughness on proliferation, differentiation, and protein synthesis of human osteoblast-like cells (MG63). Journal of Biomedical Materials Research, 1995; 29: 389-401.

23. Matge, G.: Anterior interbody fusion with the BAKcage in cervical spondylosis. Acta Neurochir 1998; 140: 18 .

24. Matge, G., Leclercq, T.A.: Rationale for interbody fusion with threaded titanium cages at cervical and lumbar levels. Results on 357 cases. Acta Neurochir 2000; 142: 425433.

25. Mayer, H.M., Wiechert Karsten, M.D.: Microsurgical Anterior Approaches to the Lumbar Spine for Interbody Fusion and Total Disc Replacement. Neurosurgery. 2002; 51: S2-159-S2-165.

26. Meier, U., Kemmesies, D.: Experiences with six different intervertebral disc spacers for spondylodesis of the cervical spine. Orthopade. 2004; 33: 1290-1299.

27. Meier, U., Grawe, A., Hajdukova, A.: Clinical Experience With Cespace, the New Intervertebral Disc Spacer by Aesculap for Spondylodesis of the Cervical Spine, in Comparison With Similar Products by Weber, Intromed, and AcroMed. Neurosurgery Quarterly. 2003; 13: 40-50.

28. Ortiz, O., Pait, T.G., Mcallister, P.: Postoperative magnetic resonance imaging with titanium implants of the thoracic and lumbar spine. Neurosurgery1996; 38: 741-745.

29. Pröbster, L., Voigt, C.H., Fuhrmann, G., Gross, U.M.: Tensile and torsional shear strength of the bone implant interface of titanium implants in the rabbit, Journal of Materials Science: Materials in Medicine, 1994; 5, 6-7: 314-319.
30. Profeta, G., De Falco, R., Ianniciello, G., Profeta, L., Cigliano, A., Raja, A.I.: Preliminary experience with anterior cervical microdiscectomy and interbody titanium cage fusion (Novus CT-Ti) in patients with cervical disc disease. Surg Neurol 2000; 53: 417-426

31. Ray, C.D.: Threaded titanium cages for lumbar interbody fusions. Spine 1997; 22: 667-680.

32. Riew, K.D., Rhee, J.M..: The use of titanium mesh cages in the cervical spine. Clin Orthop Relat Res. 2002; 4754.

33. Sawin, P.D., Traynelis, V.C., Menezes, A.H.: A comparative analysis of fusion rates and donor-site morbidity for autogeneic rib and iliac crest bone grafts in posterior cervical fusions. J Neurosurg 1998; 88: 255-265.

34. Schmieder, K., Wolzik-Grossmann, M., Pechlivanis, I., Engelhardt, M., Scholz, M., Harders, A.: Subsidence of the wing titanium cage after anterior cervical interbody fusion: 2-year follow-up study. J Neurosurg Spine. 2006 ; 4: 447-453.

35. Skolasky, R.L., Maggard, A.M., Hilibrand, A.S., Northrup, B.E., Ullrich, C.G., Albert, T.J., Coe, J.D., Riley, L.H.: $3^{\text {rd }}$. Agreement between surgeons and an independent panel with respect to surgical site fusion after single-level anterior cervical spine surgery: a prospective, multicenter study. Spine. 2006; 1; 31:E503-6.

36. Tropiano, F., Diop, A., Dejou, J.: A titanium/plasmapore coated intervertebral block to aid interbody fusion. An experimental, mechanical and histopathological study in ewes. Rivista di Neuroradiologia 1999; 12: S81-S87.

37. Tropiano, P., Bronsard, J.J., Louis, C.: Three column stabilisation through a posterior approach with titanium plasmapore intervertebral block (Prospace). Radiological and clinical study after 4 years. Rivista di Neuroradiologia 1999; 12 : S89-S94

38. Tropiano, P., Diop, A., Dejou, J., Bronsard, J.G., Poitout, D.G.: Interbody arthrodesis using a plasmapore titanium block. Mechanical and histological experimental study in sheep. Chirurgie 1999; 124: 58-65.

39. Tureyen, K.: Disc height loss after anterior cervical microdiscectomy with titanium intervertebral cage fusion. Acta Neurochir. 2003; 145: 565-569.

40. Van Jonbergen, H.P., Spruit, M., Anderson, P.G., Pavlov, P.W.: Anterior cervical interbody fusion with a titanium box cage: early radiological assessment of fusion and subsidence. Spine J. 2005; 5: 645-649.

41. Volkmann, R., Eingartner, C., Winter, E., Weller, Weise., And S.: Midterm results in 500 titanium alloy straight femoral stem prostheses -cemented and cementless technique. European Journal of Orthopaedic Surgery \& Traumatology. 1998; 133-139.

42. Wetzel, F.T., Larocca, H.: The failed posterior lumbar interbody fusion. Spine 1991; 16: 839-845.

43. Wigfield, C., Gill, S., Nelson, R., Langdon, I., Metcalf, 
N., Robertson, J.: Influence of an artificial cervical joint compared with fusion on adjacent-level motion in the treatment of degenerative cervical disc disease. J Neurosurg. 2002; 96 : 17-21.

44. Zdeblick, T.A., Ghanayem, A.J., Rapoff, A.J., Swain, C., Bassett, T., Cooke, M.E., Markel, M.: Cervical interbody fusion cages. An animal model with and without bone morphogenetic protein. Spine 1998; 23: 758-765.

Comentario al trabajo Cespace sistema de fusión intersomática cervical. Estudio preliminar en 104 casos (120 implantes) de Arregui y cols.

Los autores presentan la experiencia de un grupo español con el empleo de un implante intersomático de titanio ("CeSpace") para conseguir una artrodesis cervical. La superficie rugosa de polvo de titanio que tiene este implante le confiere una teórica mayor capacidad de osteointegración y de estabilidad, que permite colocarlo sin necesidad de añadir una estabilización complementaria con placa atornillada a los cuerpos vertebrales. La seguridad y eficacia de este implante ha sido ya demostrada por autores como Krayenbühl et al. en $2008^{1}$. A pesar de las limitaciones del presente trabajo por ser un análisis retrospectivo, el considerable número de casos tratados, con un prolongado seguimiento posquirúrgico, le confiere un importante valor descriptivo. Los resultados clínicos y de fusión radiológica obtenidos son muy similares a los de otros estudios sobre artrodesis cervical con implante intersomático metálico o biológico, si bien es de resaltar favorablemente, en el trabajo de estos autores, que no se produjo en relación con la técnica ninguna complicación de importancia. A la controversia sobre realizar una discectomía cervical sin o con artrodesis, se añade en los últimos tiempos la controversia sobre la selección del material y técnica idóneos para conseguir dicha artrodesis. No hay todavía clara evidencia en la literatura sobre la superioridad de una técnica sobre otra, y todas tienen sus ventajas e inconvenientes como bien
Arregui, R.; Aso, J.; Martínez-Quiñones, J.V.; Consolini, F.; Lambán, N.; Dominguez, M.: Cespace: sistema de fusión intersomática cervical. Estudio preliminar en 104 casos (120 implantes). Neurocirugía 2011; 22: 542-553.

Correspondencia: Arregui R. Servicio de Neurocirugía. Hospital MAZ. Zaragoza. Avda. Academia General Militar, 74. 50015. Zaragoza. e-mail: rarregui@maz.es discuten los autores en este artículo. Como objeción a las conclusiones del trabajo, cabe decir que parece arriesgada la opinión de los autores de calificar a las cajas recubiertas de titanio, similares a la empleada por ellos, como "un gold standard en la fusión cervical sin injerto óseo". En este sentido, hay que recordar que otros tipos de implantes metálicos, como por ejemplo las cajas de tantalio poroso, también han demostrado su seguridad y eficacia pero en un estudio prospectivo randomizado ${ }^{2}$, lo que supone tener un mayor nivel de evidencia del que se tiene para las cajas "CeSpace".

\section{Bibliografía}

1. Krayenbühl, N., Schneider, C., Landolt, H., and Fandino, J.: Use of an empty, plasmapore-covered titanium cage for interbody fusion after anterior cervical microdiscectomy. $\mathrm{J}$ Clin Neurosci. 2008; 15: 11-17.

2. Fernández-Fairen, M., Sala, P., Dufoo, M., Ballester, J., Murcia, A., and Merzthal, L.: Anterior Cervical Fusion With Tantalum Implant. A Prospective Randomized Controlled Study. Spine 2008; 33: 465-472

J. Ayerbe Gracia Madrid 